\title{
Programm der Jahresversammlung der DO-G. 1956 in Wien
}

Freitag, 18 . M a i

Anreise per Schiff auf der Donau ab Passau. Ankunft abends in Wien. Abholung mit Autobussen ab Hafen. Einweisung in die Quartiere durch Führer.

Sonnabend, 19. M a i

9 Uhr Eröffnung der Tagung im Auditorium maximum der Universität. Anschließend Vorträge. Mittagspause 12-15 Uhr (gemeinsames Mittagsessen im Rathauskeller).

15 Uhr Abfahrt Gruppe A mit Autobussen zum Wilhelminenberg. Führung durch die Station mit Einführungsvortrag. Anschließend Wienerwaldspaziergang. Abends evtl. Waldhornblasen auf Wienerwaldwiese. Anschließend gemeinsames Nachtmahl im Restaurant Predigtstuhl (Blick über ganz Wien).

$15 \mathrm{Uhr}$ Abfahrt Gruppe B mit Autobussen Stadtrundfahrt. Ring, Belvedere (evtl. Schönbrunn), Botanischer Garten, Höhenstraße Kobenzl-Kahlenberg (evtl. Leopoldsberg). Auf Hinfahrt kurze Besichtigung neuer städtischer Anlagen, Wienerwaldfahrt. Gemeinsames Nachtmahl am Kahlenberg (evtl. Leopoldsberg).

Pfingstson tag, 20. Mai

Wie am Sonnabend, nur Programmtausch Gruppe A-B.

Pfing stmontag, 21 . M a i

Vormittags Besuch der spanischen Hofreitschule (Vorführungen), Führungen durch Ornith. Sammlung des Naturhist. Museums, N.Ö. Landesmuseum, Hofburg (Schatzkammer). Teilnahme nach freier Wahl auch an mehreren Führungen möglich.

12-15 Uhr Mittagspause (Essen im Rathauskeller).

15-1.7 Uhr Vorträge im Auditorium maximum. Abends Oper oder Theater nach Wahl.

Di enstag, 22. Mai

Exkursion mit Autobussen Gruppe A Neusiedlersee Ostufer (Steppe, Schilfrand).

Mittwo ch, 23. M a i Gruppe B Donau-Auwälder, Marchegg.

9-12 Uhr Vorträge Audit. max., 12-15 Uhr Essen Rathauskeller.

15-17 Uhr Filmvorführungen Audit. max.

17 Uhr Abfahrt mit Autobussen nach Baden. Ankunft $18 \mathrm{Uhr}$.

19-20 Uhr Musikabend im Kursalon. 20-21 Uhr Nachtmahl. Rückfahrt 22 Uhr.

Großexkursionen nach der Tagung für speziell interessierte Ornithologen:

Reiher- und Löfflerkolonien des Neusiedlersee-Westufers ..... 3 Tage

Subalpine Vogelwelt der N.Ö. Kalkalpen $\ldots \ldots \ldots \ldots \ldots \ldots \ldots \ldots 2$ Tage

Alpine Vogelwelt der Steirischen Zentralalpen (Zirbitz) $\ldots \ldots \ldots, 4$ Tage

Untersteirisches Hügelland $\ldots \ldots \ldots \ldots \ldots \ldots \ldots \ldots \ldots \ldots \ldots, 3$ Tage

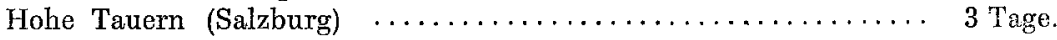

Tagungsbüro im Naturhistorischen Museum Wien 1, Burgring 7.

Tagungsort: Universität, Auditorium maximum.

Mittagessen: Rathauskeller, Neues Rathaus.

Vorträge, Vorführungen usw. wolle man bis spätestens 1. März anmelden beim 2. Vor-sitzenden der DO-G., Herrn Dr. G. Niexhammer, Bonn, Koblenzer Str. 164. 